\title{
Localized ion outflow in response to a solar wind pressure pulse
}

\author{
S. A. Fuselier, H. L. Collin, A. G. Ghielmetti, and E. S. Claflin \\ Lockheed Martin Advanced Technology Center, Palo Alto, California, USA
}

T. E. Moore and M. R. Collier

NASA Goddard Space Flight Center, Greenbelt, Maryland, USA

H. Frey and S. B. Mende
Space Sciences Laboratory, University of California, Berkeley, Berkeley, California, USA

Received 10 September 2001; revised 6 December 2001; accepted 21 December 2001; published 24 August 2002.

[1] Neutrals created by charge-exchange of low-energy $(\sim 10-100 \mathrm{eV})$ ion outflow from the ionosphere are imaged by the Low Energy Neutral Atom (LENA) imager on the IMAGE spacecraft. Comparing the neutral flux with changes in the solar wind during an ion outflow event on 24 June 2000 indicates that changes in solar wind density (and therefore dynamic pressure) are associated with episodic bursts of ion outflow. LENA images show that these bursts are confined to the duskside. Simultaneous images of the aurora from the IMAGE FUV Wideband Imaging Camera indicate that these episodic bursts on the duskside are associated with increased duskside auroral emissions. The pitch angle and charge exchange altitude for the ion outflow distributions are estimated by applying field line tracing in a model magnetic field and assuming that the outflow occurs in the auroral zone. The pitch angle information places constraints on the neutral atom images, indicating that the ion outflow observed on the duskside probably consists of high pitch angle conics and that field-aligned ion outflow from other parts of the oval cannot be observed from the IMAGE spacecraft location. INDEX TERMS: 2704 Magnetospheric Physics: Auroral phenomena (2407); 2736 Magnetospheric Physics: Magnetosphere/ionosphere interactions; 2784 Magnetospheric Physics: Solar wind/magnetosphere interactions; 2451 Ionosphere: Particle acceleration; 2407 Ionosphere: Auroral ionosphere (2704); KEYWORDS: Ionospheric outflow, solar wind interactions, auroral ionosphere, neutral particle imaging

\section{Introduction}

[2] Since the discovery of ionospheric ions in the magnetosphere during a magnetic storm [Shelley et al., 1972], the processes that allow these ions to enter the magnetosphere and the role these ions play in magnetospheric dynamics have been the subject of many investigations. Large statistical surveys of high-latitude, in situ data have demonstrated that energetic ( $>10 \mathrm{eV}$ up to several $\mathrm{keV})$ ions originate in the auroral zone ionosphere [see, e.g., Yau et al., 1984; Collin et al., 1998]. These studies have also established ion outflow dependence on season, solar cycle, and magnetospheric activity [e.g., Yau et al., 1985; Collin et al., 1998; Øieroset et al., 1999]. Statistical studies of the Earth's plasma sheet complement the studies of ionospheric ion outflow, showing that the outflow dependence on magnetospheric activity is reflected in other regions of the magnetosphere [Lennartsson, 1992].

[3] Large statistical studies of ionospheric outflow have also provided clues to the acceleration mechanisms of ionospheric ions. At energies above a few $\mathrm{eV}$, three types of ion distributions are observed. Ion beams (i.e., distribu-

Copyright 2002 by the American Geophysical Union. 0148-0227/02/2001JA000297\$09.00 tions with peak phase space density along the magnetic field) are observed at all magnetic local times with clear seasonal dependence [Collin et al., 1998]. Two types of ion conics are observed (i.e., distributions with peak phase space density at an oblique angle to the magnetic field). The first type, standard or "restricted" conics, have peak phase space density at a nearly constant pitch angle between $40^{\circ}$ and $70^{\circ}$ relative to the magnetic field (i.e., $40^{\circ}-70^{\circ}$ pitch angles in the southern hemisphere and $110^{\circ}-140^{\circ}$ pitch angles in the northern hemisphere) over the entire energy range from 0.01 to $>1 \mathrm{keV} / \mathrm{e}$ [e.g., Sharp et al., 1977; Peterson et al., 1992]. The second type, extended or "elevated" conics, have peak phase space density at pitch angles less than $30^{\circ}$ for low energies (less than $50 \mathrm{eV}$ ) and peak phase space densities nearly the same as that for standard conics at higher energies [Klumpar et al., 1984].

[4] The critical difference between the two types of conics is the finite parallel velocity and field-aligned nature of the lowest energies of the extended conics. It is believed that this finite velocity is the result of a combination of perpendicular heating and parallel acceleration of the initial ion distribution as it propagates from the ionosphere along auroral field lines [Klumpar et al., 1984; Norqvist et al., 1996; Yau and André, 1997]. Although this difference is important for understanding the physics behind the creation 
of these two types of conics, the similarity in the pitch angle distributions of conics at higher energies and the narrowness of the pitch angle distributions of all types of ion outflow are most important for the observations discussed here.

[5] Apart from the pitch angle differences at low energies, restricted and elevated conics have few other differences. Both restricted and elevated conics have occurrence probabilities that peak at pre-noon local time [Miyake et al., 1996]. The altitude dependence is different. The occurrence probability of restricted conics increases rapidly with altitude up to about $6000 \mathrm{~km}$, but levels off above this altitude. In contrast, the occurrence probability of elevated conics increases very slowly with altitude up to about $6000 \mathrm{~km}$ and then more rapidly above this altitude. The occurrence probability for elevated conics is similar to that of fieldaligned beams [Miyake et al., 1996]. Finally, there are some mass dependent features of conics, especially at energies greater than a few hundred eV [e.g., Collin and Johnson, 1985]. For the most part though, the characteristics and statistical variations of ion conics are relatively independent of ion species. The results of these statistical studies have been interpreted as evidence that elevated conics evolve out of restricted conics as the ion distributions are accelerated by parallel electric fields and heated along auroral field lines [e.g., Miyake et al., 1996].

[6] While these in situ observations have provided important clues to the origins and long term variability of ion outflow, there are some important limitations to these studies. One of the primary limitations is the timescales for accumulating "global" observations. Establishing a global picture of the ion outflow from the auroral zone using in situ measurements from a single spacecraft requires data accumulation intervals of at least 6 months. These long intervals are required because the spacecraft orbit must precess through all local times to sample the in situ outflow. With such long accumulation intervals, studying global ion outflow variations on much shorter timescales such as those for magnetic storms (days) and substorms (tens of minutes) is not possible. This limitation results from the fact that in situ outflow measurements on these timescales can establish the amount and characteristics of local outflow on a particular field line at a particular time. However, there is always the possibility that the outflow observed on one field line is different from that on adjacent field lines that are not sampled. Because of this ambiguity, the location and strength of ion outflow over substorm or storm timescales is poorly known. To be sure, there is evidence of significant outflow from the dayside cusp/cleft region, which depends on solar wind input [e.g., Moore et al., 1985]. Also, there is evidence, or at least the inference of substantial outflow from the nightside and other regions of the auroral oval associated with storms and substorms [e.g., Shelley, 1985].

[7] The launch of the IMAGE spacecraft provides a unique opportunity to address the issue of global ion outflow on timescales shorter than months. One of the instruments on the spacecraft is a novel low energy neutral atom (LENA) imager [Ghielmetti et al., 1994; Moore et al., 2000], which, in principle, can produce a global image of the ion outflow with a cadence of $2 \mathrm{~min}$. In addition to this imager, there is a suite of Far Ultraviolet (FUV) imagers which produce simultaneous global images of the Earth's aurora.
[8] The purpose of this paper is to investigate the ionospheric outflow on a global scale using IMAGE observations from a single event. This event was chosen because the solar wind input to the magnetosphere changed during the imaging interval. The ionospheric response to the changes in the solar wind input is investigated using images from the LENA imager. FUV observations of the aurora and a magnetospheric magnetic field model are used to guide the interpretation of these images. In particular, they are used to qualify statements regarding the variations in the location and intensity of the outflow over the measurement period.

\section{Instrumentation}

[9] The LENA imager uses a tungsten surface to convert low energy neutral atoms into negative ions [Moore et al., 2000]. These negative ions are subsequently accelerated and analyzed in a time-of-flight mass spectrometer. In principle, the imager can determine the mass, energy, and direction of arrival of neutral atoms created by charge exchange of ionospheric ions with the Earth's atmosphere and geocorona. For the interval of interest, energy resolution within the energy passband of the imager was not possible. However, the passband of the imager is relatively narrow (between a few $\mathrm{eV}$ and about $150 \mathrm{eV}$ ), so the inability to determine energy does not represent a significant limitation for observations of ion outflow in this paper.

[10] The unique nature of the LENA imager design results in some special contributions to background counts in the instrument [Ghielmetti et al., 1994]. Ultraviolet light (from scattered sunlight in the geocorona, dayglow on the sunlit side of the Earth, and aurora at high latitudes) can cause production of electrons within the instrument. Furthermore, this light can cause photo-attachment of electrons to neutrals in the ambient gas near the conversion surface of the instrument. These negative ions will be accelerated and analyzed by the time-of-flight mass spectrometer. Electrons are also accelerated and produce background starts and stops in the mass spectrometer.

[11] Another source of background is low and high energy ion sputtering. High-energy (several keV) neutrals impinging on the conversion surface will sputter low energy neutrals and ions. A small fraction of these will be negatively charged and will be accelerated in the ion optics and analyzed by the time-of-flight mass spectrometer. Energetic neutral $\mathrm{O}$ can sputter $\mathrm{H}^{-}$and $\mathrm{O}^{-}$and other ions with high electron affinity from the surface. With lower efficiency, the more abundant energetic neutral $\mathrm{H}$ can sputter $\mathrm{H}^{-}$and $\mathrm{O}^{-}$ and other ions from the conversion surface as well [Moore et al., 2000]. Thus, sputtering will create mass peaks in the time-of-flight mass spectrum that may not correctly represent the incident low-energy neutral population. Lower energy neutrals incident on the surface can also produce sputtered negative ions. As a result of high and low energy sputtering, the composition of the incident neutral beam is not easily identified unless the observed signal is predominantly $\mathrm{H}^{-}$or $\mathrm{O}^{-}$.

[12] Finally, the LENA imager has a collimator and charged particle rejecter, which rejects ions up to about $100 \mathrm{keV} / \mathrm{e}$. However, very energetic charged particles, such as those found in the radiation belts, can penetrate the 


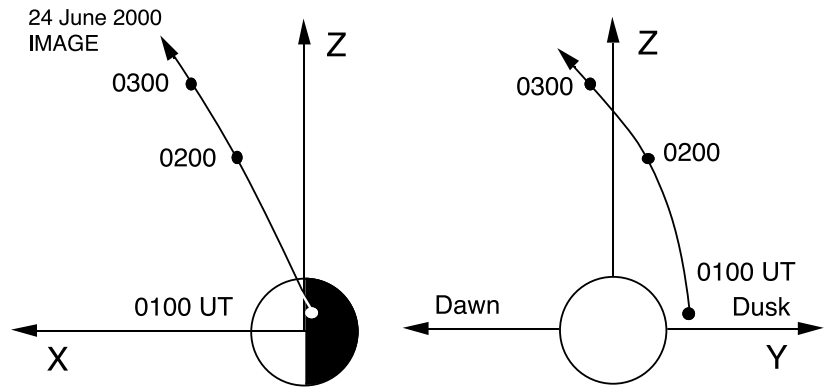

Figure 1. Perspective views of the IMAGE spacecraft orbit on 24 June 2000 . The spacecraft starts in the radiation belts at about 0100 UT. As the altitude increases, the spacecraft has good view of the duskside, high-latitude ionosphere.

instrument and create background in the mass spectrometer. As long as the spacecraft is outside the radiation belts, the double-coincidence detection scheme in the time-of-flight mass spectrometer in LENA reduces the background sufficiently to detect neutrals from ion outflow [e.g., Moore et al., 2001; Fuselier et al., 2001]. The best observations of ion outflow are obtained from the polar cap region relatively near the Earth (within $\sim 4 \mathrm{R}_{\mathrm{E}}$ altitude) because this location maximizes the outflow signal and minimizes background from charged particles.

[13] The Far Ultraviolet (FUV) imagers on the IMAGE spacecraft provide global images of the Earth's electron and proton aurora. Here, auroral images in the wavelength range between 140 and $180 \mathrm{~nm}$ (produced by precipitating electrons and protons) from the Wideband Imaging Camera (WIC) are used for context measurements and to determine the pitch angles of the arriving neutrals in the LENA instrument. These auroral images, like the neutral atom images, are obtained at the spacecraft spin rate of 2 minutes. However, since the auroral imagers look at the Earth only, the exposure time for each image is of the order of 5 seconds. In addition to the WIC images, proton aurora images from the Spectrographic Imager (SI12) are used to identify the cusp footprint [e.g., Frey et al., 2002; Fuselier et al., 2001].

\section{Observations}

[14] Figure 1 shows perspective views of the IMAGE spacecraft location on 24 June 2000 from 0100 to 0300 UT. At $0100 \mathrm{UT}$, the spacecraft was at the dusk terminator, at low altitudes, and near the equator. The spacecraft exited the outer magnetosphere at high latitudes and entered the polar cap at about 0110 UT. In this region, the energetic ion flux was low, resulting in low background from this source. As the spacecraft moved toward apogee, it viewed of the northern hemisphere high latitudes from the duskside, sunward of the terminator.

[15] Figure 2 shows a summary of the solar wind conditions on 24 June 2000 and the ionospheric outflow response to these solar wind conditions. Solar wind observations were obtained from the Comprehensive Plasma Instrument [Frank et al., 1994] and the Magnetic Field Experiment [Kokubun et al., 1994] on the Geotail space- craft. This solar wind monitor was located near the subsolar region (approximately 17, 1.5, $-2.5 \mathrm{R}_{\mathrm{E}}$ GSE). The top panel shows the solar wind density, the second panel shows the solar wind velocity, and the third panels shows the $Z_{\mathrm{GSE}}$ component of the solar wind magnetic field. The bottom panel shows the total countrate in the earthward looking sector of the LENA imager field-of-view (on the IMAGE spacecraft). The solar wind data from the upstream monitor have been convected to the ionosphere (using a convection time of $5 \mathrm{~min}$ ) and an additional delay (discussed below) was added to account for the time required for charge exchanged neutrals from the ionospheric outflow to arrive at the IMAGE spacecraft.

[16] The solar wind magnetic field was mainly northward during the interval with a brief southward excursion at around 0110 UT. The velocity was relatively constant, especially during the period of high solar wind density from 0120 to 0215 UT. At the start of this high solar wind density interval, the solar wind velocity decreased, indicating that the high solar wind density interval from 0120 to 0215 UT was not associated with an interplanetary shock. Rather, the density increases are interpreted here as solar wind pressure pulses. Each jump in density (e.g., at 0120 UT, 0145 UT, and 0150 UT) results in a jump in

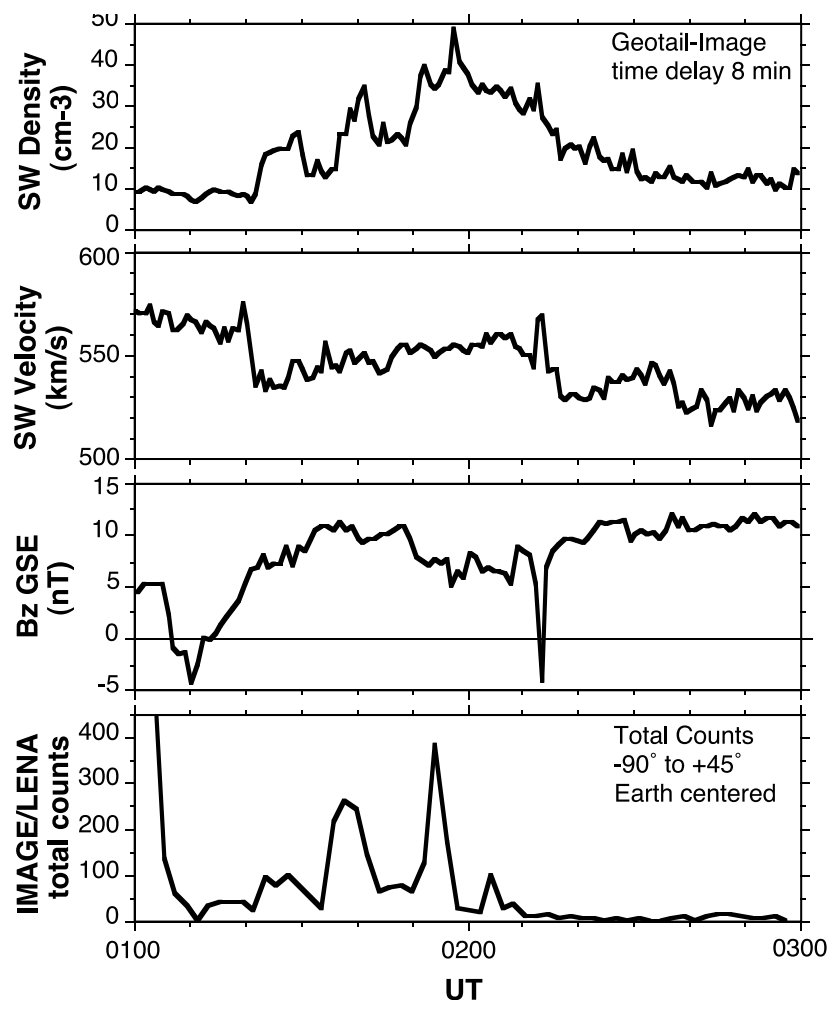

Figure 2. Solar wind plasma and magnetic field observed from an upstream monitor compared to the ionospheric response observed from the IMAGE spacecraft. Solar wind observations are convected to the ionosphere as discussed in the text. The solar wind density increases in the top panel are correlated with ion outflow pulses in the bottom panel. The solar wind velocity and magnetic field are relatively constant during these solar wind density increases, indicating that these are likely pressure pulses in the solar wind. 


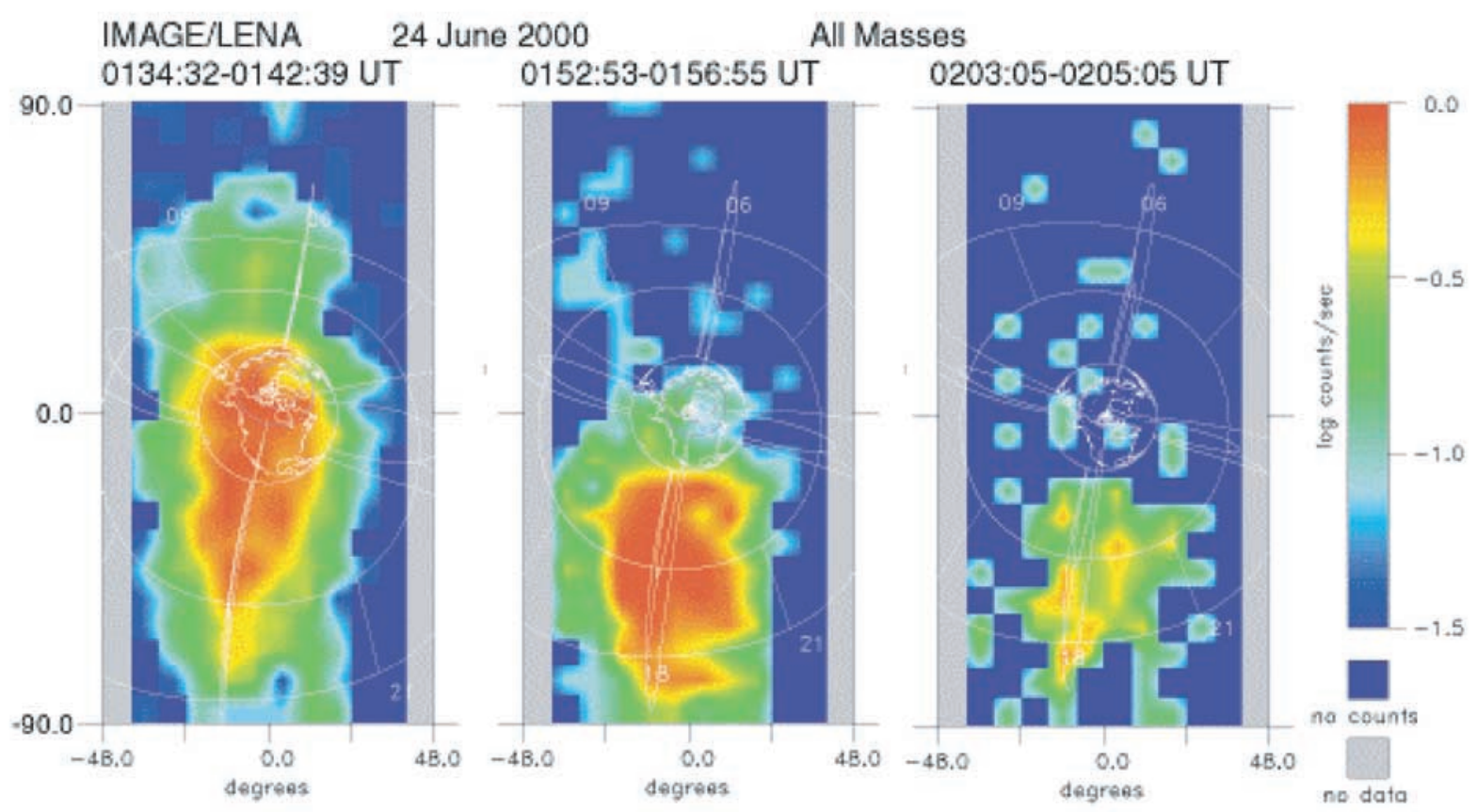

Figure 3. Images of ion outflow from the LENA imager (summed over all masses). The three times correspond to three bursts of outflow seen in Figure 1. In each of the images, the Earth is at the center and the perspective is from the duskside. Clockwise from the left-hand side of each image, magnetic field lines at noon, dusk, midnight, and dawn are shown for L values of 3 and 5. Ion outflow occurs mainly on the duskside and, in each successive image, with increasing distance from the Earth.

dynamic pressure that compresses the magnetosphere. However, because the IMF is northward, the increased dynamic pressure does not necessarily result in increased magnetospheric activity.

[17] Increases in the ionospheric outflow appear to be associated with density changes in the solar wind. Prior to 0110 UT, the IMAGE spacecraft was at low latitudes and in a high background region where it could not image the ionospheric outflow. However, after 0110 UT, there are three distinct pulses in the ionospheric outflow associated with the three pulses of solar wind density in the top panel of Figure 2. A fourth pulse of ionospheric outflow at 0205 UT occurs just after the peak in the solar wind density at 0157 UT. Other details of the ion outflow after 0200 are discussed below.

[18] Although Figure 2 suggests that there may be a good correlation between density changes in the solar wind and ion outflow from the ionosphere, timing issues between the upstream monitor and the ion outflow observations introduce some ambiguity. The $5 \mathrm{~min}$ delay time between the observation of the solar wind pressure pulses and the estimated time of their arrival in the ionosphere (estimated from the solar wind propagation to the bow shock, through the magnetosheath, and along a magnetic field line into the ionosphere) is uncertain by about $1-2 \mathrm{~min}$. An additional 3 min delay was imposed on the Geotail observations to account for the propagation time of neutrals between the ionosphere and the IMAGE spacecraft. This delay time was determined by assuming that the outflow consisted of $50 \mathrm{eV}$ ionospheric $\mathrm{H}^{+}$that was charge exchanged at high altitudes (see below) and propagated to the spacecraft altitude of about $3 \mathrm{R}_{\mathrm{E}}$. This delay time also has an uncertainty of a few minutes since the energy of the ionospheric outflow population is only known to be in the energy bandpass of the instrument (between a few $\mathrm{eV}$ and $150 \mathrm{eV}$ ). Thus, the precise timing of the ionospheric outflow with the arrival of the solar wind pressure pulses in the ionosphere is uncertain by several minutes. However, the relative spacing of the three solar wind pulses and the three ion outflow pulses and the reasonable assumptions for the absolute timing strongly suggests that these phenomena are related.

[19] Figure 3 shows LENA images of three outflow pulses centered at about 0140, 0155, and 0205 UT. In each image, the perspective view is from the IMAGE spacecraft with the Earth at the center. Magnetic field lines are shown at L shells of 3 and 5 in the sunward (left, 1200 local time), duskward (down, 1800 LT), tailward (right, 2400 LT), and dawnward (up, 0600 LT) directions. The ionospheric outflow is seen mainly in the duskward direction at about 1800 LT. In each successive image, ionospheric outflow pulses move further off the Earth direction and decrease in intensity. After the last image in Figure 3, the ionospheric outflow was essentially absent (see Figure 2).

[20] One of the important capabilities of the IMAGE instrument is its ability to determine the mass of the arriving neutral. As noted in section 2, there are some instrumental effects that must be considered in determining the species of the ion outflow. Although all masses (integrating over the total time-of-flight spectrum) are shown in Figure 3, for these three images, the mass resolved data (not shown) 


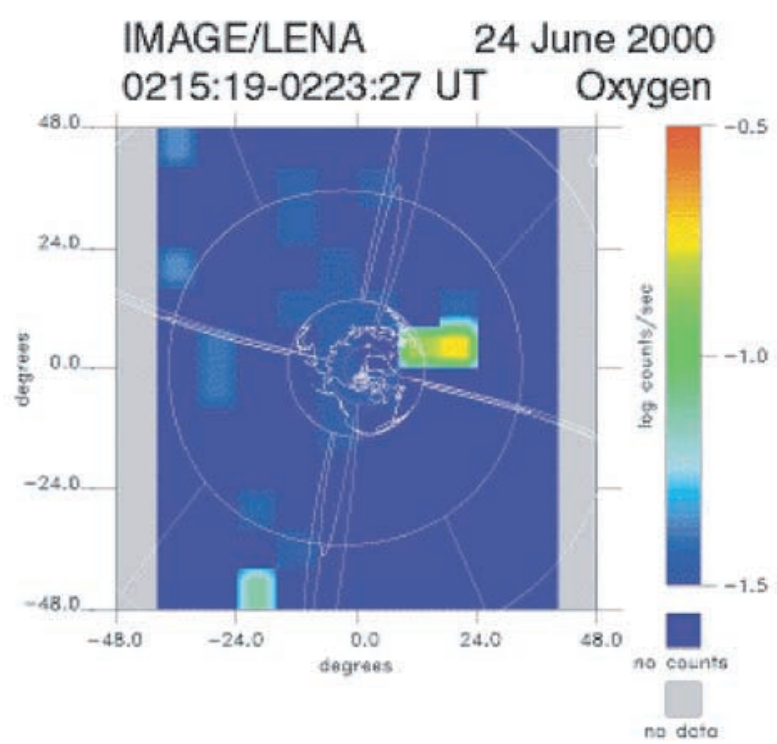

Figure 4. Oxygen outflow image from LENA. The format is the same as in Figure 3; however, the perspective has shifted to a location nearly over the geographic pole due to spacecraft motion. A weak outflow is seen in the early morning near the Earth.

indicated that the neutral $\mathrm{H}$ countrate was comparable to the neutral $\mathrm{O}$ countrate. Thus, the neutral signal in the images in Figure 3 probably contained significant neutral $\mathrm{H}$ from charge-exchanged $\mathrm{H}^{+}$outflow. As discussed in the section 2 , sputtering off the tungsten conversion surface in the instrument could cause some of the residual neutral $\mathrm{O}$ signal.

[21] Figure 4 shows a neutral $\mathrm{O}$ image taken from 0215 to 0223 UT. During this interval, the neutral H countrate in was very low. Thus, the image in Figure 4 is likely neutral $\mathrm{O}$ from charge exchanged $\mathrm{O}^{+}$outflow. At this time, the spacecraft was at more than $4 \mathrm{R}_{\mathrm{E}}$ altitude. Although the $\mathrm{O}$ signal is relatively weak, it was clearly absent from images before and after the time interval in Figure 4 (not shown). Assuming that the energy of the original $\mathrm{O}^{+}$outflow was $50 \mathrm{eV}$, a different delay time is required to determine what part of the solar wind density profile was associated with this outflow. Because the spacecraft is further away from the Earth and $50 \mathrm{eV} \mathrm{O}^{+}$(and $50 \mathrm{eV}$ neutral $\mathrm{O}$, after charge exchange) is propagating at a speed that is 4 times slower than $50 \mathrm{eV} \mathrm{H}$, the delay time from the ionosphere to the spacecraft is between 16 and 18 minutes. This time delay indicates that Oxygen started in the ionosphere as $\mathrm{O}^{+}$ between 0200 and 0202 UT in Figure 2. Therefore, this Oxygen outflow is associated with the period just after the largest density pulse in the solar wind.

[22] Figure 5 shows three images of the aurora from the FUV/WIC imager. Each image was transformed into invariant latitude-magnetic local time (MLT) coordinates with the sun direction at the top. Background dayglow was removed from these images. This background correction affects auroral emissions between about 0800 and 1600 MLT. These images correspond to the center times of the three bursts of ionospheric outflow in Figure 3 (again, allowing for a $3 \mathrm{~min}$ propagation time between the ionosphere and the spacecraft for $50 \mathrm{eV} \mathrm{H}$ ). In the first auroral image in Figure 5, the complete auroral oval is not within the $15^{\circ}$ field of view of the WIC imager because the IMAGE

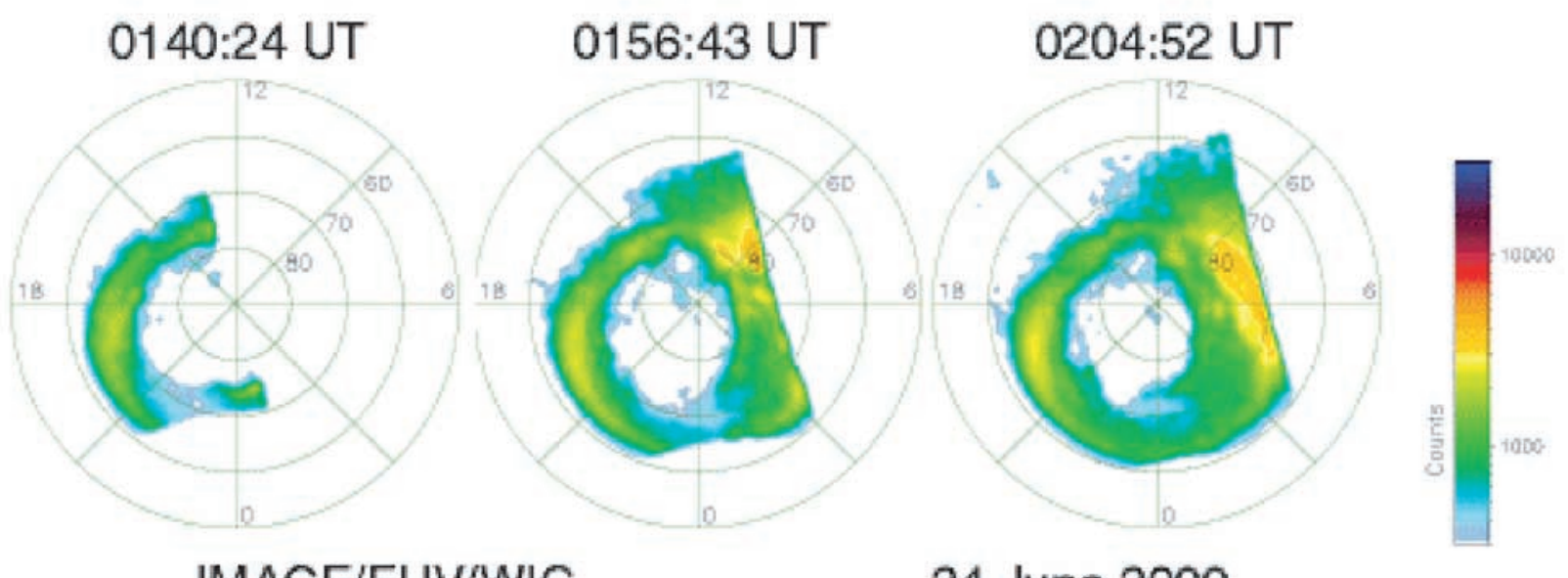

IMAGE/FUV/WIC

24 June 2000

Figure 5. Auroral images $(140-160 \mathrm{~nm})$ corresponding to the center times of the three ion outflow bursts in Figure 3. In each image, the emissions were transformed into the MLT-Invariant Latitude coordinate system. In the first image, only the part of the oval from about 1300 to 0100 UT was in the field of view of the auroral imager. By the third image, nearly the entire oval was in the field of view. On the duskside, emissions peak near 1900 UT. In each successive image, the duskside emissions increase in intensity. However, the second and third images show that the auroral emissions were more intense on the dawnside. The spot at $82^{\circ}$ latitude and 11 MLT in the third image is the ionospheric footprint of the cusp. 
IMAGE/WIC

24-Jun-2000 (00176) Time: 020400.0

Tilt Angle: 15.4 deg

Dst: $-6 \mathrm{nPa}$
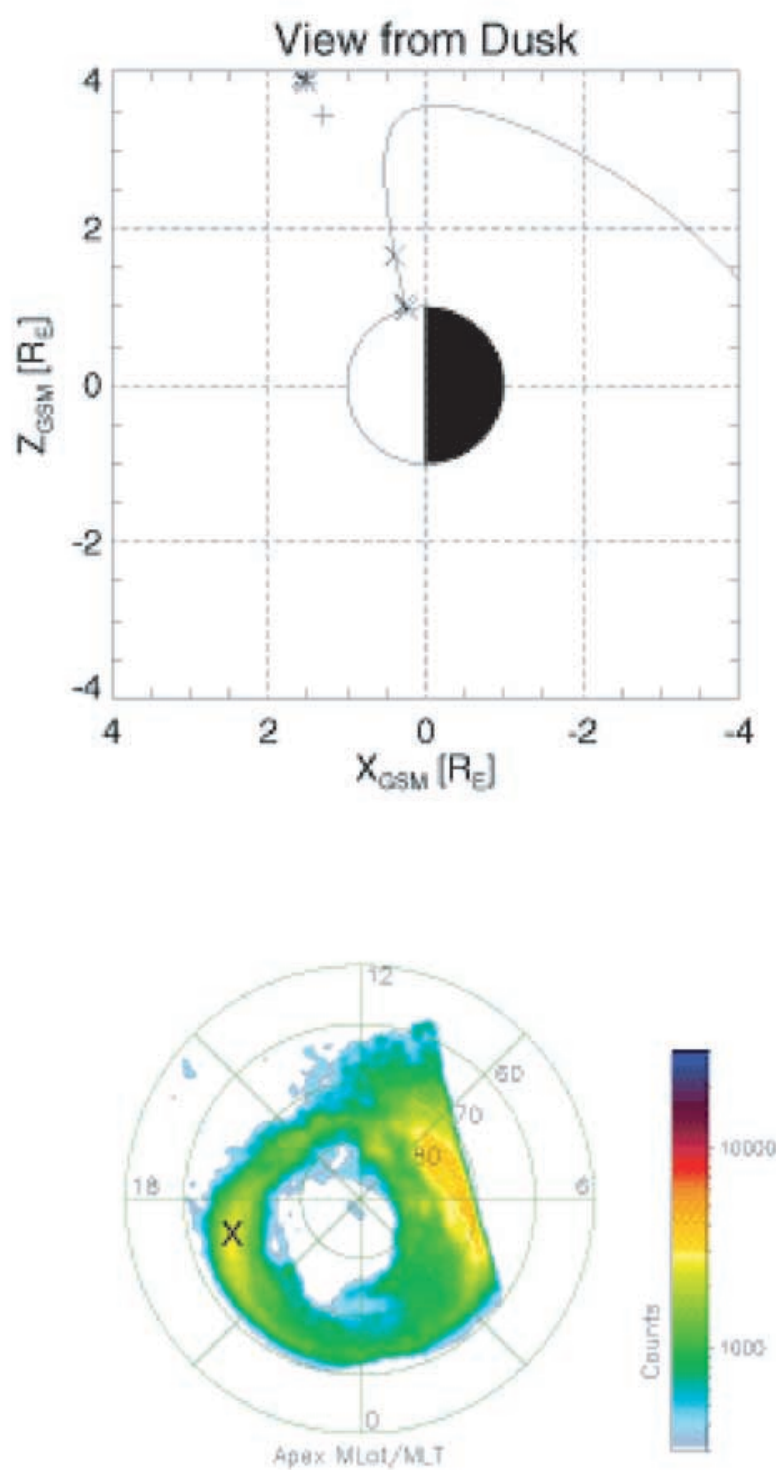

\section{WIND}

Time: 015733.2

DynP: $15.3 n T$ Bswx: $-4.4 n T$

Bswy: $-5.7 n T$ Bswz: $6.8 \mathrm{nT}$
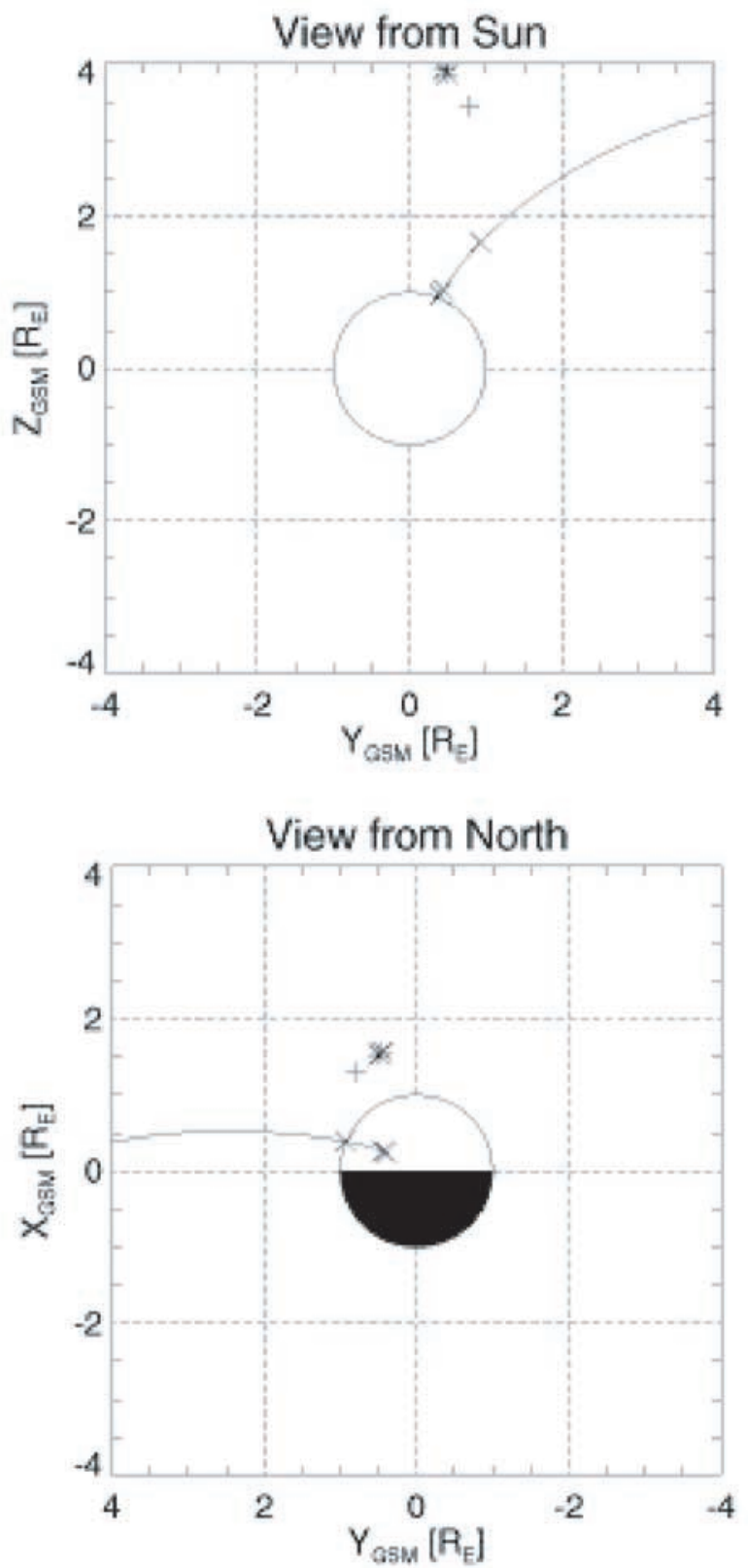

Figure 6. Magnetic field line traced from the third auroral image in Figure 5. The field line is traced from the peak flux in the auroral image in the lower left hand corner. Three perspective views are shown of this magnetospheric field line. In each view, x's marked along the field line show the location of 500, 1000 , and $6000 \mathrm{~km}$ altitudes. The asterisk shows the location of the IMAGE spacecraft at the time the auroral image was taken.

spacecraft was at low altitude. For this first image, the oval between about 1300 and 0100 MLT was visible. As the spacecraft altitude increased, more of the auroral oval was visible, as is evident in the subsequent images in Figure 5.
[23] The aurora in Figure 5 at 0140 UT (corresponding to the pulse of ionospheric outflow in Figure 3 starting at the same time) contains a relatively broad peak of emissions centered at about 1900 MLT. In subsequent images, this 
peak persists and brightens by about 30\%. After 0140 UT, when the field-of-view encompasses nearly the full auroral oval, Figure 5 shows that the aurora is much more intense on the dawnside between 0300 and 0900 MLT when compared to the duskside emissions. Auroral emissions at 1200 and 2400 MLT are comparatively weaker than those on the dawn and dusk sides. The image at 0204 UT in Figure 5 contains a spot at about $82^{\circ}$ latitude and 11 MLT. A simultaneous image from the SI12 imager (not shown) also contains a spot in the same location, indicating that this spot is associated with proton precipitation. Consistent with other high solar wind dynamic pressure intervals, the spot at 11 MLT is interpreted as the footprint of the cusp [e.g., Frey et al., 2002; Fuselier et al., 2001]. Its location is consistent with the negative By component of the solar wind magnetic field (not shown).

[24] Using the fact that ionospheric outflow is associated with the auroral oval [e.g., Collin et al., 1998], the auroral images in Figure 5 are used to determine the pitch angles and charge exchange altitudes of the ion outflow. Figure 6 illustrates the procedure. Shown are 3 perspective views of a magnetic field line traced in the Tsyganenko-96 magnetic field model. This field line was traced from the peak emissions at 1900 MLT in the auroral image at 0204 UT. The field line tracing was done from this MLT (indicated by the $\mathrm{x}$ on the auroral oval image in the lower left-hand part of Figure 5) assuming $100 \mathrm{~km}$ altitude for the auroral emissions. Three x's on the field line in the perspective views of the magnetic field lines show 500, 1000, and $6000 \mathrm{~km}$ altitudes. To be detected by the LENA imager, ions are assumed to propagate along the field line, charge exchange at one of these altitudes, and propagation in a straight line to the IMAGE spacecraft after charge exchange. Ionospheric ions originating from 1900 MLT and executing this charge exchange process would have pitch angles of $137^{\circ}, 135^{\circ}$, and $133^{\circ}$, at charge exchange altitudes of 500,1000, and $6000 \mathrm{~km}$, respectively. Since the magnetic field is directed into the northern ionosphere, these angles represent upflowing ions with angles of $\sim 45^{\circ}$ relative to the magnetic field at the time of charge exchange. Neutrals produced by charge exchange of ions that had pitch angles larger or smaller than about $130^{\circ}-140^{\circ}$ would not be detected by the LENA imager because their trajectories would not intersect the IMAGE spacecraft location.

[25] The neutral atom image in Figure 3 at 0203 UT shows substantial flux well off the Earth direction. Charge exchange products from near the Earth $(\sim 1000 \mathrm{~km}$ altitude $)$ would appear more in the Earth direction in the LENA image. Therefore, the charge exchange altitude for the ion outflow that LENA imaged in Figure 3 was probably of the order of $1 \mathrm{R}_{\mathrm{E}}$ or greater. Figure 7 shows the pitch angles for ions that originate in the auroral zone and whose charge exchanged products have trajectories that intersect the IMAGE spacecraft. Plotted is pitch angle as a function of local time for the three images in Figure 3, assuming that ionospheric ions charge exchange at $6000 \mathrm{~km}$. The uncertainties in the pitch angles indicate the range of pitch angles for the latitudes when the aurora flux was significant (e.g., between $61^{\circ}$ and $70^{\circ}$ invariant latitude on the duskside in Figure 5).

[26] Figure 7 shows that the LENA imager views a relatively narrow range of pitch angles for the ion outflow

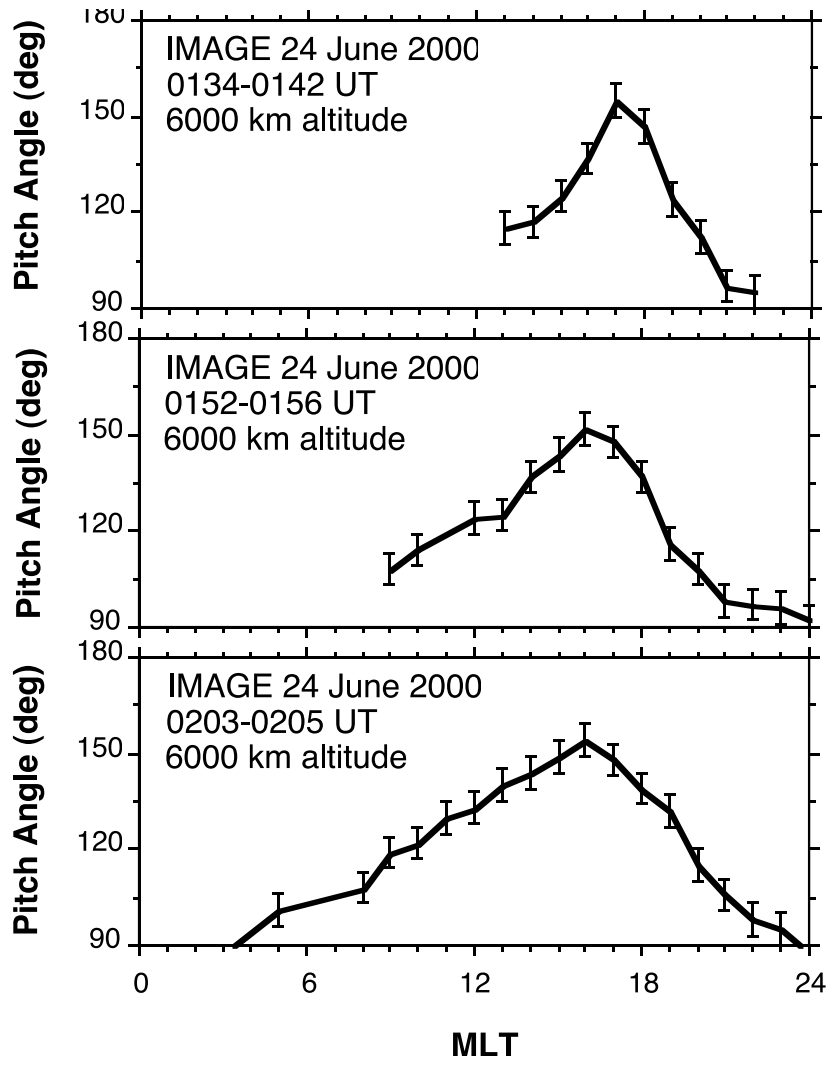

Figure 7. Pitch angles of ion outflow from the auroral zone that charge exchange at $6000 \mathrm{~km}$ altitude. The three panels correspond to the three auroral images and three ion outflow images in Figures 5 and 3, respectively. Pitch angles were determined from field line tracings like the one in Figure 6. Error bars show the range of pitch angles determined from the latitudinal extent of the auroral emissions in the FUV images. At 1800 MLT, the ion outflow in Figure 3 comes from $120^{\circ}-145^{\circ}$ pitch angle ions that have charge exchanged at about $6000 \mathrm{~km}$ altitude. For MLT between 0 and about 6 , the LENA imager was not measuring ion outflow because the observed pitch angles are less than $90^{\circ}$. Over the rest of the oval, the flux seen by the imager is strongly dependent on the observed pitch angle.

out of the auroral zone at any given MLT. Furthermore, continuous charge exchange along the magnetic field line does not improve the range of pitch angles that the LENA imager views because the observable pitch angle is only weakly dependent on charge exchange altitude. Figure 7 also shows that measured pitch angles between 0 and 6 MLT are less than $90^{\circ}$, indicating that no outflow can be imaged from the duskside vantage point of the IMAGE spacecraft. Finally, at 1200 and 2400 MLT, imaging is limited to ion outflow distributions that have substantial flux near $130^{\circ}$ and $90^{\circ}$, respectively.

\section{Discussion}

[27] LENA images in this paper show that episodic bursts of ion outflow are likely associated with increases in the solar wind density (and therefore dynamic pressure). The 
outflow is "prompt" in the sense that timing differences between the pressure pulses observed by the upstream monitor and the arrival of neutral flux at the IMAGE spacecraft can be accounted for completely by propagation of the solar wind signal to the ionosphere and immediate response of the ionosphere to this signal. As pointed out in section 3 , the timing is uncertain by a few minutes between the arrival of solar wind disturbances in the ionosphere and the arrival of neutrals created by charge exchange of ion outflow in response to these disturbances. However, the relative timing and duration of solar wind density enhancements and bursts of neutral flux and the reasonable assumptions for the absolute timing strongly suggests that the events are related (see Figure 2). The changes in the outflow are large, varying by a factor of 5 or more over time periods of several minutes (assuming $50 \mathrm{eV}$ Hydrogen neutrals charge-exchanged from $\mathrm{H}^{+}$outflow). Peak fluxes are $\sim 10^{8}\left(\mathrm{~cm}^{2} \mathrm{~s}\right)^{-1}$; however, as discussed below, the actual outflow flux is probably strongly pitch angle dependent and, since all pitch angles are not measured simultaneously, the absolute outflow rate is difficult to determine. Nonetheless, the peak flux is higher than but compares favorably with typical ion outflow fluxes of $10^{7}-10^{8}\left(\mathrm{~cm}^{2} \mathrm{~s}\right)^{-1}$ [e.g., Yau et al., 1983]. All of these variations occur during an extended period of northward IMF, when the magnetospheric activity is relatively low and reasonably constant. While changes in the outflow are likely caused by the solar wind dynamic pressure variations, the exact coupling between these variations and ion outflow is not known at present.

[28] Combining the fact that ion outflow occurs in the auroral zone [e.g., Collin et al., 1998] with simultaneous images of the far-ultraviolet aurora during the episodic ion outflow events, the pitch angles observed by the LENA imager for a charge exchange altitude of $6000 \mathrm{~km}$ is shown in Figure 7. Comparing Figure 7 with the images in Figure 3, it is apparent that the peak neutral flux comes from ion outflow at pitch angles between $125^{\circ}$ and $145^{\circ}$. This approximate pitch angle range coincides very well with that of restricted conics or the higher energy part of extended conics in the northern hemisphere. Both of these conic distributions have very narrow pitch angle distributions which peak between $120^{\circ}$ and $140^{\circ}$ at or above about 50 eV [Peterson et al., 1992; Miyake et al., 1996].

[29] Previous in situ observations show that occurrence frequencies of these conics peak on the dawnside. In contrast, the episodic outflow events imaged by LENA are limited to the duskside (see Figure 3). This apparent discrepancy is removed by considering the measured pitch angles as a function of MLT in Figure 7.

[30] Because restricted conics and extended conics at higher energies have such narrow pitch angle distributions, the vantage point of the IMAGE spacecraft during this outflow event is not suitable for imaging these types of ion outflow distributions on the dawnside. In fact, Figure 7 shows that the duskside vantage point does not allow imaging of any charge-exchanged ion outflow between 0000 and 0600 MLT. Pitch angles greater than $135^{\circ}$ are not viewed at magnetic local times earlier than 1200 MLT for any of the three outflow images. It is true that pitch angles of about $135^{\circ}$ are observed in two places on the auroral oval. The first is around 1800 MLT and the second is around 1300 MLT. However, the images in Figure 5 show that the aurora was somewhat weaker near noon MLT compared to the duskside around 1800 MLT. Finally, Figure 7 also shows that the LENA images in Figure 3 are restricted to high pitch angle distributions over the entire oval. Therefore field-aligned ion beams (with peak flux near $180^{\circ}$ pitch angles), and the low energy end of extended conics (also with peak flux at about $180^{\circ}$ pitch angles) are not imaged. Thus, for this event, the relative contributions of different parts of the auroral oval to the total ion outflow cannot be determined.

[31] This result is particularly important for observing outflow from the cusp/cleft. The image at 0204 UT shows that the cusp footpoint was located at $82^{\circ}$ latitude and 11 MLT. Only high pitch angle ions are observable from this part of the auroral zone. Thus, field-aligned outflow from the cusp is not detectable for this event.

[32] There is one exception to this lack of observed flux at local times other than $\sim 1800$ MLT. Figure 4 shows a short burst of neutral $\mathrm{O}$ that is associated with the time period just after the peak in the solar wind density in Figure 2 (when the propagation time between the ionosphere and the spacecraft for $50 \mathrm{eV}$ Oxygen is taken into account). The flux is relatively weak and Figure 7 shows that these ions probably had pitch angles near $90^{\circ}$ when they charge exchanged at relatively low altitudes. Because the range of pitch angles that can be viewed is limited, it cannot be determined whether the low flux is caused by weak outflow or by a viewing geometry that is limited to the extreme pitch angles of an otherwise intense outflow event that peaks at smaller pitch angles. Thus, even the total outflow of the flux that is imaged is not easily determined.

[33] In summary, episodic and prompt ion outflow events are associated with increases in solar wind dynamic pressure. The changes in ion outflow that are observed over timescales of minutes are not associated with changes in the IMF $B_{z}$ component, nor are they associated with enhancements in magnetospheric activity. By combining knowledge of ion outflow distributions with simultaneous observations of the aurora, the LENA images are placed into context with previous in situ measurements of ion outflow. For this event, the LENA imager probably observes restricted conics or the higher energy part of extended conics on the duskside. These restricted or extended conics are closely associated with enhanced auroral emissions.

[34] Acknowledgments. The IMAGE mission is the result of work by a large number of dedicated scientists and engineers. Dr. J. L. Burch is the PI of the IMAGE mission. Solar wind data were provided by the Geotail through the NSSDC. The authors thank Dr. S. Kokubun, PI of the Geotail magnetometer experiment and Dr. L. A. Frank, PI of the Geotail Comprehensive Plasma Instrument.

[35] Janet G. Luhmann thanks M. Yamauchi and another referee for their assistance in evaluating this paper.

\section{References}

Collin, H. L., and R. G. Johnson, Some mass dependent features of energetic ion conics over the auroral regions, J. Geophys. Res., 90, 9911, 1985.

Collin, H. L., W. K. Peterson, O. W. Lennartsson, and J. F. Drake, The seasonal variation of auroral ion beams, Geophys. Res. Lett., 25, 4071, 1998.

Frank, L. A., K. L. Ackerson, W. R. Paterson, J. A. Lee, M. R. English, and G. L. Pickett, The comprehensive plasma instrumentation (CPI) for the GEOTAIL spacecraft, J. Geomagn. Geoelectr., 46, 23, 1994.

Frey, H. U., S. B. Mende, T. J. Immel, S. A. Fuselier, E. S. Claflin, J.-C. 
Gerard, and B. Hubert, Proton aurora in the cusp, J. Geophys. Res., 107(A7), 10.1029/2001JA900161, 2002.

Fuselier, S. A., et al., Ion outflow observed by IMAGE: Implications for source regions and heating mechanisms, Geophys. Res. Lett., 28, 1163, 2001.

Ghielmetti, A. G., E. G. Shelley, S. A. Fuselier, F. Herrero, M. F. Smith P. Wurz, P. Bochsler, and T. Stephen, Mass spectrograph for imaging low energy neutral atoms, Opt. Eng., 33, 362, 1994.

Klumpar, D. M., W. K. Peterson, and E. G. Shelley, Direct evidence for two-stage (bimodal) accelerations of ionospheric ions, J. Geophys. Res., 89, 10,799, 1984

Kokubun, S., T. Yamamoto, M. H. Acuña, K. Hayashi, K. Shirokawa, and H. Kawano, The Geotail magnetic field experiment, J. Geomagn. Geoelectr., 46, 7, 1994

Lennartsson, O. W., A scenario for solar wind penetration of Earth's magnetic tail based on ion composition data from the ISEE 1 spacecraft, J. Geophys. Res., 97, 19,222, 1992.

Miyake, W., T. Mukai, and N. Kaya, On the origins of the upward shift of elevated (bimodal) ion conics in velocity space, J. Geophys. Res., 101, 26,961, 1996.

Moore, T. E., C. R. Chappell, M. Lockwood, and J. H. Waite Jr., Superthermal ion signatures of auroral acceleration processes, J. Geophys. Res. 90, 1611, 1985

Moore, T. E., et al., The low-energy neutral atom imager for IMAGE, in The IMAGE Mission, edited by J. L. Burch, pp. 155-195, Kluwer Acad., Norwell, Mass., 2000

Moore, T. E., et al., Low energy neutral atoms in the magnetosphere, Geophys. Res. Lett., 28, 1143, 2001.

Norqvist, P., M. André, L. Eliasson, A. I. Eriksson, L. Blomberg, H. Lühr, and J. H. Clemmons, Ion cyclotron heating in the dayside magnetosphere, J. Geophys. Res., 101, 13,179, 1996.

Øieroset, M., M. Yamauchi, L. Liszka, and B. Hultqvist, Energetic ion outflow from the dayside ionosphere: Categorization, classification and statistical study, J. Geophys. Res., 104, 24,915, 1999.
Peterson, W. K., H. L. Collin, M. F. Doherty, and C. M. Bjorklund, $\mathrm{O}^{+}$and $\mathrm{He}^{+}$restricted and extended (bi-modal) ion conic distributions, Geophys. Res. Lett., 19, 1439, 1992.

Sharp, R. D., R. G. Johnson, and E. G. Shelley, Observations of an ionospheric acceleration mechanism producing energetic $(\mathrm{keV})$ ions primarily normal to the geomagnetic field direction, J. Geophys. Res., 82, 3324, 1977.

Shelley, E. G., Circulation of energetic ions of terrestrial origin in the magnetosphere, Adv. Space Res., 5, 401, 1985.

Shelley, E. G., R. G. Johnson, and R. D. Sharp, Satellite observations of energetic heavy ions during a geomagnetic storm, J. Geophys. Res., 77, 6104, 1972.

Yau, A. W., and M. André, Sources of ion outflow in the high latitude ionosphere, Space Sci. Rev., 80, 1, 1997.

Yau, A. W., B. A. Whalen, A. G. McNamara, P. J. Kellogg, and W. Bernstein, Particle and wave observations of low-altitude ionospheric ion acceleration events, J. Geophys. Res., 88, 341, 1983.

Yau, A. W., W. B. Whalen, W. K. Peterson, and E. G. Shelley, Distribution of upflowing ionospheric ions in the high-altitude polar cap and auroral ionosphere, J. Geophys. Res., 89, 5507, 1984

Yau, A. W., P. H. Beckwith, W. K. Peterson, and E. G. Shelley, Long-term (solar cycle) and seasonal variations of upflowing ionospheric ion events at DE 1 altitudes, J. Geophys. Res., 90, 6395, 1985.

E. S. Claflin, H. L. Collin, S. A. Fuselier, and A. G. Ghielmetti, Lockheed Martin Advanced Technology Center, 3251 Hanover Street, O/L9-42, Building 255, Palo Alto, CA 94304-1191, USA. (fuselier@spasci.com)

M. R. Collier and T. E. Moore, NASA Goddard Space Flight Center, Greenbelt, MD 20771, USA.

H. Frey and S. B. Mende, Space Sciences Laboratory, University of California, Berkeley, Berkeley, CA 94720-7450, USA. 